zoologists, already taken action in several cases. It has, for example, recommended, but not yet passed, the suspension of the rules in the case of Holothuria versus Physalia (Opinion 76), and is at the moment preparing to adjudicate on the name of the common house-fly (see NATURE, January 27, p. II 5).

The Commission has also--urged thereto by its devoted secretary, Dr. C. W. Stiles-attempted to draw up for various groups lists of agreed and unalterable names, Nomina conservanda.

If, owing to the war and the peace, so thorough a worker as $\mathrm{Mr}$. Chapman can have, apparently, forgotten or remained ignorant of the Commission's work, there must be many in the rising generation to whom it is equally unknown. If they cannot find what they want in the Report of the International Congress of Zoologists or, more accessibly, in the American periodical Science and in the Smithsonian "Miscellaneous Collections," they may like to know that the present members of the Commission in this country are Dr. Hartert and Dr. Jordan of Tring Museum, Dr. W. E. Hoyle of the Welsh National Museum, and myself at the Natural History Museum ; also that the Commission is seeking to fill one of its vacancies with an Australasian representative.

$$
\text { F. A. Bather. }
$$

\section{The Formation of Coloured Bows and Glories.}

WHEN favourably situated, a person may see rings of coloured light round the shadow of his own head, as cast upon a neighbouring fog-bank or cloud. These coloured rings or glories, as they are called, have been explained by previous writers as merely coronas due to particles near the surface of the cloud scattering light reflected from deeper portions of the cloud ; in other words, the effect is regarded as of the same nature as the ordinary corona but due to secondary scattering. That this explanation cannot be accepted as correct is definitely shown by experimental observations made with artificial clouds.

The experimental arrangement is the same as that used by Mecke (Ann. der Phys., vol. 6I), and if the eye of the observer be placed on the same side of the cloud chamber as the source, so as to look down very nearly along the path of the beam passing through it, a succession of colours is seen along its track through the cloud. These colours also change as the angle of observation is changed; and the smaller the particle the greater is the angle from which they can be seen. The complete system of rings is obtained on illuminating the cloud with a beam of sunlight, and may be viewed in a perpendicular direction with the aid of a plane sheet of glass held at $45^{\circ}$ in front of the cloud chamber, so that the observer's head does not screen the cloud chamber from the illuminating pencil. The observations prove that the phenomenon under discussion is shown by every position of the cloud, and therefore really arises from primary scattering by the droplets of water.

That the glories or brocken-bows arise in a way which is quite different from that of the ordinary transmission coronas is proved by the fact that the sequence of colours in the brocken-bows and in the transmission coronas due to cloud particles of the same size are far from being identical. The normal corona, due to larger drops, shows a central white field with a brownish-red edge, which is surrounded by the familiar coloured rings, but in the brocken-bows the arrangement is different and varies somewhat with the size of the drop. It is sometimes found that just round the central spot (which is the image of the source of light reflected from the first surface of the observing flask) there is a distinct minimum of intensity exhibiting colour; then the intensity increases, the colour being greenish-white bordered by a brownish-red edge, and then follows the usual succession of coloured rings as in the coronas. It is sometimes found that round the central spot there is a clear maximum, and then a belt of minimum intensity, and then again a maximum; in other words, there is an oscillatory distribution of intensity ; in the central field of the brocken-bow only red and green rings or belts are present in different intensities, whitishyellow colour being totally absent, while in the corresponding coronal rings the central field is yellowish-white or nearly without colour.

In order to understand how the glories are formed, we have to consider the light which travels back towards the source from the droplets. This arises in two ways : (a) by reflection from the front surface of the droplets; $(b)$ by two refractions and one internal reflection. When a plane wave falls on the spherical particles and is reflected back at its external surface, the reflected wave front is strongly divergent, and, as a result, it merely adds a little to the general illumination of the field and does not give rise to any notable diffraction effect. But the wave front $(b)$ formed by internal reflection is not so divergent as in $(a)$, and is limited by a cusped edge, at which it is doubled back. When the droplet is small the path differences between back and front of the wave near the cusped edge are very small. Hence we may, without appreciable error, consider the wave front to be a simple spherical cap of appropriate radius. As a sufficient approximation, we may assume the centre of this spherical cap to be the image of a point placed on the axis at an infinite distance, produced by two refractions and one reflection. We have to find in directions making a small angle with the axis back towards the direction of the primary source the aggregate effect of this wave cap. The problem now is the same as the diffraction produced by a small circular opening in the screen on which light is propagated in spherical waves from a point source. We take as the axis of symmetry the line drawn from the source to the centre of the opening, and it is required to find the intensity of illumination at any point of a plane screen parallel to the plane of the opening and at a distance from the later. The detailed mathematical treatment of this problem is given in Gray and Mathews's "Treatise on Bessel Functions and their Applications to Physics," chapter xiv., and the result is applied in this case for the measurements of the positions of the maximum and minimum in the glory-rings. Considering the experimental difficulties and assumption in the theory, the results agree fairly well with the observations.

B. B. Ray.

University College of Science and Technology, 92 Upper Circular Road, Calcutta, December I3.

\section{The Definition of Limiting Equality.}

In teaching the calculus to students of applied mathematics and physics I have found that the definitions of limiting value and of limiting equality given in practically all our text-books are unsatisfactory, and in my opinion inadequate. According to these books the test of limiting equality of two magnitudes is that their difference shall become less than any assignable quantity, however small. But this condition is satisfied by any two quantities whatever if they vanish simultaneously, and it affords no justification for the use of statements such as $d y=f^{\prime}(x) d x$; on the other hand, if the quantities remain finite in the 